\title{
TECNOLOGIAS NO ENSINO DE GEOMETRIA MOLECULAR
}

\section{TECHNOLOGIES IN MOLECULAR GEOMETRY TEACHING}

\author{
João Ribeiro Franco Neto', Rejane Maria Ghisolfi da Silva1 \\ Recebido para publicação em 04/06/2008 \\ Aceito para publicação em 15/06/2008
}

\section{RESUMO}

Este estudo teve como objetivo investigar a utilização de diferentes tecnologias no ensino de Geometria Molecular e, fundamentalmente, identificar se o software ChemSketch ${ }^{\circledR}$ potencia ou desfavorece a elaboração de modelos mais adequados de estruturas químicas. Para subsidiar este trabalho, recorreu-se à pesquisa qualitativa, que se consolidou em um Estudo de Caso. O estudo de caso foi o método de procedimento adotado, voltado para uma turma de 28 alunos da $2^{\mathrm{a}}$ série do Ensino Médio, de uma escola particular, do estado de Minas Gerais. Os alunos tiveram oportunidade de vivenciar diferentes situações de ensino de Química, com tecnologias, e também de avaliar sobre seus aspectos relacionados à aprendizagem em Química. Os dados foram coletados por meio de questionários; gravações, em áudio e vídeo, das atividades; elaboração de um planejamento das atividades de Química, envolvendo as tecnologias: quadro e giz, bolas de isopor e varetas e o software ChemSketch ${ }^{\circledR}$. As análises foram feitas segundo duas perspectivas: (i) avaliação dos alunos a respeito da utilização de tais tecnologias e (ii) observações e registros realizados nos ambientes de aprendizagem. A partir dessas análises, pôde-se perceber que o desenvolvimento das atividades de forma combinada entre tecnologias favorece a aprendizagem para os educandos, visto que, desse modo, eles constroem as estruturas de forma mais adequada. Embora o software proporcionasse uma visualização melhor das estruturas das moléculas, os alunos não obtiveram um desempenho satisfatório, o que sugere que, na utilização do computador, o professor desempenha um papel fundamental.

Palavras-chave: Tecnologias. Química. Geometria Molecular.

\begin{abstract}
The main aim of this study was to investigate the use of different technologies in molecular geometry teaching. Moreover, it aims to identify if the software ChemSketch ${ }^{\circledR}$ facilitates or hinders the elaboration of more adequate models of chemical structures. The study took a qualitative approach in the form of a case study. The subjects of the study were 28, second year high school students from a private school in the state of Minas Gerais, Brazil. The students took the opportunity
\end{abstract}

\footnotetext{
1 Universidade Federal de Uberlândia
} 
to deal with different situations during their chemistry classes which involved the use of technologies, and also to evaluate aspects related to the learning of chemistry. The data was collected through questionnaires, audio and video recording of activities, and the planning of chemistry lab activities involving technology and resources such as: chalkboard and chalk, Styrofoam ball, sticks and the software ChemSketch ${ }^{\circledR}$. The data analysis was done from two perspectives: (i) students' evaluation concerning the use of technology, and (ii) the observations and records in the learning environments. The analysis demonstrated that the development of the activities using technologies in a balanced way supports students as they build the structures in a more adequate way. Although the software provided a better view of the molecules structures, the students did not have a satisfactory performance, which suggests that the teacher performs a fundamental role when students use computer to study chemistry.

Keywords: Technologies. Chemistry. Molecular geometry.

\section{Introdução}

Em um mundo submetido ao impacto dos meios de comunicação e da alta tecnologia, a escola se vê desafiada a redefinir seus objetivos e suas práticas pedagógicas. Desse modo, os professores são confrontados de várias maneiras perante às demandas da contemporaneidade que exigem "que a escola proponha dinâmicas pedagógicas que não se limitem a transmissão ou disponibilização de informações, inserindo nessas dinâmicas as tecnologias de informação e comunicação, de forma as reestruturar a organização curricular fechada e as perspectivas conteúdistas que vem caracterizando-a”. (BONILLA, 2005, p. 91). Nesse contexto, como professor de Ciências e Química do ensino básico em escolas públicas e particulares há mais de 24 anos, tenho vivenciado os inúmeros esforços em todos os níveis de intervenção no contexto escolar, incluindo órgãos e instâncias políticas para introduzir as tecnologias de informação e comunicação na educação. E foram as próprias exigências vindas da sociedade atual aliadas a minha preocupação com a melhoria do ensino de Ciências/Química que começaram a impor-me uma necessidade de mudança nos processos de ensino e aprendizagem. Nesse sentido, mesmo inseguro diante do novo, ousei a incorporar a informática nos processos de ensino e aprendizagem. Via nos recursos computacionais possibilidades de transformar a prática pedagógica criando novas situações de aprendizagem que fos- sem mais significativas para os alunos. Entendia que era "inegável a necessidade de introduzir novas tecnologias na educação, até mesmo como forma de questionamento do paradigma tradicional de ensino ainda hegemônico no contexto educativo" (SILVA, 2005, p. 33). Para isso a preocupação primeira foi com minha formação, pois tinha os computadores em uma das escolas em que atuava, mas como fazer com que essa ferramenta apoiasse minhas atividades de ensino?

As aprendizagens sobre geometria molecular são importantes visto que os estudantes de química apresentam dificuldades em relacionar a fórmula molecular, as características da molécula e estrutura geométrica (JOHNSTONE, 1991; GABEL; BUNCE, 1994). Nesse sentido, é necessário que eles desenvolvam habilidades específicas para visualizar moléculas no espaço, facilitando a resolução de exercícios em Química Geral e principalmente em Química Orgânica (BARNEA; DORI, 1999; DORI, 1995; DORI; HAMEIRI, 1998). Para isto é necessário que a representação seja dinâmica, podendo desse modo aprimorar a visualização tridimensional. (SEDDON; SHUBBER, 1985; SEDDON; MOORE, 1986; TUCKEY et al., 1991).

Muitos estudantes também demonstram dificuldades em relacionar as combinações orgânicas (BROOK, 1988; RYLES, 1990; SCHMIDT, 1992; SHANI; SINGERMAN, 1982; SIMPSON, 1983). Nesse sentido, pesquisadores têm apresentado diferentes abordagens instrucionais como apoio ao 
ensino de Química, adaptando estratégias de ensino baseadas na mudança conceitual (KRAJCIK, 1991), integrando atividades práticas com a sala de aula (JOHNSTONE; LETTON, 1990), utilizando modelos concretos (COPOLO; HOUNSHELL, 1995) е o emprego das novas tecnologias como ferramentas de aprendizado (BARNEA; DORI, 1999; KOZMA, RUSSEL; JONES; MARX; DAVIS, 1996; WU, KRAJCIK; SOLOWAY, 2001).

A utilização de modelos concretos aliados às novas tecnologias produzem uma nova ferramenta de aprendizagem promissora (WU; KRAJCIK; SOLOWAY, 2001).

De acordo com Taber (1997), existem concepções alternativas que podem ser adquiridas por experiência do dia-a-dia com o manuseio de objetos. Assim, elas apresentam uma dificuldade maior em ser modificadas. No que se refere às ligações químicas, devido ao seu grau de abstração, a dificuldade com o aluno aumenta, pois os mesmos não têm idéia sobre a forma como os átomos se ligam, dificultando a sua aprendizagem.

Segundo Marcelo Giordan, “as representações dessas partículas submicroscópicas, cujo meio de veiculação pode variar desde o papel, passando pelos conjuntos plásticos, isopor e madeira, chegando à tela do computador ou à projeção holográfica. Varia-se o meio e também as formas de representação, nesse caso com o objetivo de destacar uma ou outra propriedade da molécula”. (GIORDAN, 2005, pg. 290).

As representações a que se refere o autor são fundamentalmente os modelos atômicos e moleculares utilizados para tornar possível a visualização de idéias complexas, processos e sistemas (BARNEA; DORI, 2000).

Desse modo, algumas perguntas me inquietavam: como vem se dando a prática dos professores no ensino de Geometria molecular? Como favorecer uma aprendizagem mais significativa e efetiva para os alunos? Quais as tecnologias que podem ser exploradas em uma aula de Geometria Molecular? Quais são as dificuldades e facilidades em optar por esta ou aquela tecnologia? Será que a utilização do

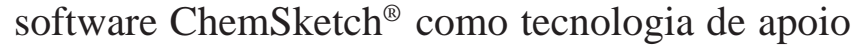
ao ensino ajudará os alunos a compreenderem me- lhor o tópico geometria molecular? Quais são as possibilidades na utilização dessa tecnologia? Em que difere de outras tecnologias?

Frente a tantas perguntas e inquietações, logo surge a necessidade de encontrar alternativas de mudanças em busca da criação de situações de ensino que proporcionem aos alunos uma melhor compreensão sobre o tema em estudo.

\section{Objetivo e método de estudo}

O objetivo geral do estudo foi investigar a utilização de diferentes tecnologias no ensino de Geometria Molecular e, fundamentalmente, identificar se o software ChemSketch ${ }^{\circledR}$ potencia ou desfavorece a elaboração de modelos mais adequados de estruturas químicas.

Para subsidiar este trabalho, recorreu-se à pesquisa qualitativa, que se consolidou em um Estudo de Caso. O estudo de caso foi o método de procedimento adotado, voltado para uma turma de 28 alunos da $2^{\text {a }}$ série do Ensino Médio, de uma escola particular, do estado de Minas Gerais.

Foram realizadas entrevistas com seis professores do Ensino Médio das escolas das redes de ensino municipal, estadual e particular da cidade de Ituiutaba - MG, buscando desenhar o perfil dos professores, suas habilidades com as novas tecnologias e a forma como eles ensinam o tópico pesquisado. Apresentava 45 questões que tinha por objetivo conduzir e ordenar as alternativas em termos de sua importância relativa.

Com base no que faziam os professores do ensino médio nas aulas de Química e em pesquisa realizada sobre como ensinar o tema foram escolhidas três (3) tecnologias de apoio ao ensino para serem desenvolvidas na turma pesquisada, sendo que as atividades propostas foram executadas de acordo com a tabela 1 .

Os compostos utilizados nas três atividades foram os mesmos, conferindo assim maior confiabilidade aos dados obtidos. Para o desenvolvimento do trabalho investigativo organizamos três situações de ensino sobre a mesma temática em três lugares diferentes. As tecnologias quadro e giz foram uti- 
Tabela 1 - Programação das atividades da pesquisa

\begin{tabular}{|c|c|c|c|}
\hline & $\begin{array}{c}\text { Quadro } \\
\text { e giz }\end{array}$ & $\begin{array}{c}\text { Bolas } \\
\text { de Iso- } \\
\text { por }\end{array}$ & $\begin{array}{c}\text { Software } \\
\text { ChemSke- } \\
\text { tch }^{\circledR}\end{array}$ \\
\hline Grupo A & $1^{\circ}$ & $2^{\circ}$ \\
\hline Grupo B & & $1^{\circ}$ & $2^{\circ}$ \\
\hline Grupo C & & $2^{\circ}$ & $1^{\circ}$ \\
\hline Grupo D & $2^{\circ}$ & & $1^{\circ}$ \\
\hline
\end{tabular}

lizadas na sala de aula, as bolas de isopor no laboratório de química e o software ChemSketch ${ }^{\circledR}$ no laboratório de tecnologia educacional da escola.

O questionário foi usado com o objetivo de obter informações diretamente do aluno, através de um formulário, com 25 questões, visando delinear um perfil desse aluno e verificar suas habilidades com as novas tecnologias. Esse procedimento, realizado com 28 alunos que integravam o grupo a ser pesquisado, configurou-se como um dos instrumentos essenciais para a investigação.

As gravações em vídeo dos estudantes pretenderam registrar as imagens de como os alunos realizavam as atividades propostas. Foram prejudicadas pela dificuldade em capturar a imagem de vários alunos ao mesmo tempo.

As fotografias registraram a montagem dos modelos tipo "bola-vareta" - e foram de fundamental importância para investigar os resultados obtidos na análise com as bolas de isopor.

No laboratório de informática o software ScreenCam ${ }^{\circledR}$ capturou e registrou o desenvolvimento da atividade pelo aluno. A vantagem do uso deste recurso é a possibilidade da observação completa das ações dos usuários, propiciando uma avaliação melhor das limitações e enganos do software que está sendo utilizado. Isso permite uma visão mais aprofundada da aplicação, e não só dos relatos do usuário.

Com isso é possível complementar e confrontar a informação colecionada por outros instrumentos. Em outras palavras, os dados do questionário podem ser confirmados ou até mesmo poderão ser negados pelos dados registrados durante a observação.

A relevância deste estudo reside na possibilidade de contribuir para a reflexão sobre as diferentes tecnologias que podem ser exploradas no ensino de Química, muitas vezes pautado pelo modelo de transmissão e recepção, tendo como tecnologia somente quadro e giz.

Este trabalho constituiu tema de dissertação do Mestrado em Química da UFU - Universidade Federal de Uberlândia, realizada no ano de 2007.

\section{Modelagem}

Uma forma de pensar sobre modelos é como instrumentos que relacionam a teoria com a suposta realidade.

Segundo Levine (1974), modelo pode ser definido como uma contribuição, uma ferramenta específica para compreender conceitos mais abstratos. Ingham e Gilbert (1991) apresentam modelo como representação simplificada de um fenômeno no qual concentra a atenção em aspectos específicos e facilita o pensamento científico.

Conforme Hardwicke (1995), modelo é uma representação de um objeto ou de um conceito. A ciência busca investigar e representar o mundo natural; para isso recorre à modelagem, tornando-se um dos aspectos centrais da ciência. Nesse sentido, a palavra "modelo" pode ter vários e diferentes usos.

Sant'anna (2002. pg. 509) conceitua modelagem molecular como "investigação das estruturas e das propriedades moleculares usando a química computacional e as técnicas de visualização gráfica visando fornecer uma representação tridimensional, sob um dado conjunto de circunstâncias”.

Na esteira do debate, Santos (2001. pg. 5) relata o domínio de atuação da modelagem molecular referindo-se "a aplicação de modelos teóricos para representar e manipular a estrutura de moléculas, estudar reações químicas e estabelecer relações entre a estrutura e propriedades da matéria constituem”.

Segundo Gilbert e Boulter (1995), o modelo pode ser compreendido como uma representação parcial de uma idéia, objeto, processo ou fenômeno para um determinado sistema. A utilização de modelos e modelagem no ensino tem sido um tema constante de pesquisa na área de educação 
(GILBERT; BOULTER, 2000).

Tiberghien (1994) dedicou-se a avaliar as formas e os resultados de modelização no ensino de ciências. Nersessian (1995) questiona até que ponto o conhecimento das práticas de pensamento dos cientistas pode auxiliar na elaboração de estratégias pedagógicas. Essas pesquisas demonstraram o papel exercido pelos modelos na aprendizagem de conteúdos científicos.

\subsection{Representação em 2D e 3D}

Segundo Gasteiger (2003), a representação 2D (bidimensional) é o idioma universal natural dos químicos. Para o autor estes diagramas de estrutura são modelos e são projetados para fazer as moléculas mais concebíveis. Neles, os átomos são caracterizados pelos símbolos e os elétrons unidos valendo-se de linhas. Porém o diagrama da estrutura química é incompleto, pois simplifica a representação da molécula ao demonstrar somente átomos unidos e o tipo de ligação existente.

A representação 3D (tridimensional) apresenta uma quantidade maior de informações como a posição dos átomos no espaço, o ângulo e a distância entre eles na construção da molécula.

Gasteiger (2003) afirma que no início foram dados nomes a determinadas combinações para que pudessem ser caracterizadas. Na maioria dos casos eram nomes usuais que ainda estão em uso. Assim passaram a ser utilizados símbolos para abreviar esses nomes, criando uma classificação sistemática de combinações. A teoria da estrutura e novas técnicas experimentais trouxeram um entendimento melhor para a estrutura da molécula, combinando os diagramas de estruturas famosas e o arranjo em 3D das moléculas.

Uma das metas mais importantes na utilização de softwares de modelagem é que ele deve representar as estruturas químicas e transferir os vários tipos de representação para os softwares, facilitando a visualização da estrutura.

São introduzidas quatro informações básicas: nomenclatura usual, nomenclatura sistemática, anotação química e matemática para as estruturas químicas a serem estudadas.

\subsection{Tipos de modelagens}

As representações mais utilizadas em química como referência em publicações ou livros didáticos são as modelagem stick (vareta), balls e stick (pau e bola) e spacefill.

Segundo Milagres (2001), o modelo "pau e bola” pode ser ótimo modelo de ensino, pois permite que os alunos "observem” os átomos. O problema é que essas representações são bidimensionais, dificultando a visualização tridimensional pelos alunos.

De acordo com Whitten (2003), os modelos de "pau e bola" usam bolas de cores diferentes para representar átomos e traços para representar as ligações.

Também conhecida como fórmula estrutural plana ou fórmula estrutural de Couper, ela demonstra as ligações entre os elementos, sendo cada par de elétrons entre dois átomos representado por um traço. Indica os átomos, a proporção entre eles, bem como as ligações covalentes existentes, que podem ser assim representadas como estrutural completa e condensada.

\subsection{Softwares de modelagem em química}

Atualmente, muitos pesquisadores (ANJOS; GIORDAN, 2004; ANJOS, 2004; WU et al, 2001; KIBOSS, 2002; CHANG, 2001; EICHLER; DEL PINO, 2006) têm se dedicado a estudar a importância e os resultados da utilização de ferramentas computacionais em ensino e aprendizagem de Química.

Na Educação Química, nos últimos dez anos, houve um verdadeiro salto na produção de softwares educacionais, abrangendo diversas áreas do conhecimento químico.

Para desenvolver a pesquisa foram analisados alguns softwares de química que pudessem auxiliar no desenvolvimento da temática Geometria Molecular, tais como: BKchem ${ }^{\circledR}$, ChemDraw ${ }^{\circledR}$, ChemDraw Ultra ${ }^{\circledR}$, ChemDraw Pro ${ }^{\circledR}$, HyperChem $7^{\circledR}$, Hyperchem Lite ${ }^{\circledR}$, Pocket Hyperchem ${ }^{\circledR}$, Pocket HyperChem $^{\circledR}$, Isis Draw ${ }^{\circledR}$, ChemWindow ${ }^{\circledR}$, AIM2000 ${ }^{\circledR}$, Chem 4-D ${ }^{\circledR}$ e ChemSketch ${ }^{\circledR}$. 
O ChemSketch ${ }^{\circledR}$ foi escolhido como instrumento de análise por ser um software gratuito e por conter interface compatível com a maioria de editores de texto e com a Internet. É um software desenvolvido pela ACD/LABS - Advanced Chemistry Development, Inc. para ser rodado na plataforma Windows ${ }^{\circledR}$ que pode ser baixado da Internet por professores ou alunos e usado para construir equações químicas, estruturas moleculares, esquemas de aulas práticas com determinada facilidade. Ele incorpora avançadas características como a capacidade de girar moléculas no espaço, visualizar moléculas de diferentes maneiras e tem uma versão comercial incompatível com a versão livre. A versão livre, usada na pesquisa, é liberada para professores e estudantes.

Além da estrutura plana, o ChemSketch ${ }^{\circledR}$ possui um módulo de geração e visualização de moléculas em $3 \mathrm{D}$, que é o $\mathrm{ACD} / 3 \mathrm{D}$ Viewer $^{\circledR}$, que apresenta várias formas de representação de uma estrutura química: Wireframe (tipo de representação 3D que mostra a molécula na forma de "linhas") , Sticks (tipo de representação 3D que mostra a molécula na forma de “varas”), Balls \& Sticks (tipo de representação 3D que mostra a molécula na forma “bolas e varas”), Spacefill (tipo de representação 3D que mostra os espaços vazios da molécula "preenchidos" (semelhante ao modelo Stuart), Dots Only (tipo de representação 3D que mostra "somente pontos” representando os átomos e ligações da molécula), Disks (tipo de representação que mostra os átomos na forma de discos, muito semelhante à forma Spacefill, porém sem o efeito 3D), With Dots (mostra os "pontos” da representação 3D em qualquer forma de visualização), With Dots combinando com Balls \& Sticks.

\subsection{Geometria molecular - VSEPR}

A geometria molecular é um parâmetro de importância fundamental para a previsão da polaridade de uma molécula. Esta, por sua vez, permite inferir sobre o tipo e intensidade das interações intermoleculares que se podem estabelecer entre moléculas no composto puro, ou com átomos, ou moléculas de outras substâncias. Contudo, a pre- visão da geometria molecular, até de moléculas simples, representa freqüentemente um problema que muitos alunos do ensino médio e, por vezes do superior, não conseguem superar (BIRK; KURTZ, 1999; FURIO; CALATAYUD, 1996). Estas dificuldades estão usualmente relacionadas com a suposta necessidade de determinar, previamente, a estrutura de Lewis (ou a fórmula estrutural) para as moléculas.

A geometria tridimensional das moléculas é determinada pela orientação relativa de suas ligações covalentes. A idéia do modelo VSEPR foi primeiro explorado pelos químicos ingleses Nevil Sidgwick e Herbert Powell em 1940.

Sidgwick e Powell sugeriram ser possível prever a forma aproximada de uma molécula com base no número de pares de elétrons na camada de valência do átomo central, no caso de moléculas que contém somente ligações simples. O seu método de previsão tem como base a minimização da repulsão dos pares de elétrons, isto é, a orientação dos orbitais deve ser tal que as distâncias entre elas sejam o maior possível.

Em 1957, o químico Ronald Gillespie, baseando-se em trabalhos prévios de Sidgwick e Powell, criou uma ferramenta muito simples para prever a geometria das moléculas, tendo uma maior exatidão na geometria molecular.

A teoria recebeu o nome de Valence Shell Electron Pair Repulsion Theory (VSEPR) ou Teoria de Repulsão dos Pares Eletrônicos de Valência e se baseia em um simples argumento de que os grupos de elétrons se repelem uns com os outros e a forma adotada pela molécula será aquela em que a repulsão dos grupos eletrônicos seja mínima (GILLESPIE (2004); GILLESPIE; ROBINSON (1996)).

Segundo Russel (2006), um erro comum cometido por quem usa o método VSEPR é confundir a orientação espacial de um conjunto de pares de elétrons com a forma molecular ou geometria da molécula.

\section{Resultados e discussões}

Acompanhando muito de perto o cotidiano 
da escola e, em particular, da turma investigada, foi possível entender que a experiência vivida foi fundamental no processo de produção do conhecimento sobre os diferentes modos de abordagem que podem ser utilizados no ensino de Geometria Molecular. O professor/pesquisador compartilhou as atitudes dos alunos, suas reações, dificuldades e avanços.

Os alunos teoricamente aprendem, por exemplo, que os átomos se unem por ligações covalentes para formar moléculas. Porém, têm dificuldades de representar ou prever a geometria dessas moléculas. A utilização de modelos, por sua vez, pode promover a elaboração de várias e úteis interpretações, explicações, bem como formas de compreensão e de previsão que envolve a aplicação de modelos teóricos na descrição de estruturas e propriedades de interesse da Química.

Desse modo, tomou-se, pois, como ponto de partida, a criação de diferentes situações de ensino, valendo-se do que usualmente é usado no ensino de Geometria molecular no ensino médio na escola.

Assim, tais tecnologias foram objeto de investigação e de reflexão crítica, com refinamentos e possibilidades de reinterpretações, na perspectiva de analisar qualitativamente as aprendizagens com a utilização de diferentes tecnologias de apoio ao ensino e, fundamentalmente, identificando se o software ChemSketch ${ }^{\circledR}$ potencia ou desfavorece a elaboração de modelos mais adequados de estruturas químicas.

A proposta de trabalho se fundamenta no pressuposto de que os modelos são, por sua natureza, uma forma de manifestação do pensamento teórico (revelação do concreto em forma de conceitos com a mediação do pensamento). Desse modo, o modelo, e a representação se constituem em resultados de uma complexa atividade cognitiva que inclui, fundamentalmente, a elaboração mental do objeto, a expressão concreta em imagens das relações essenciais da realidade que não são captadas sensorialmente. Assim, o modelo construído não é algo pronto e consolidado, mas processo que vai se formando e se transformando, e é este saber representacional do aluno que se constituiu em desafio, no sentido de captá-lo, resgatá-lo e sistematizá-lo, identificando e colocando a descoberto um conhecimento acerca do pensamento teórico-conceitual que vai se constituindo e que, conseqüentemente, pode ser promotor de desenvolvimento e aprendizagens.

Para o desenvolvimento da atividade, a turma de alunos foi dividida aleatoriamente em quatro grupos denominados pelas letras A, B, C, D, sendo que cada grupo teria oito participantes. Foi-lhes dito, nessa ocasião, que as aulas transcorreriam com trabalhos em grupo e que eles teriam oportunidade de mudar de grupo se assim o desejassem. O professor/ pesquisador apresentou para os alunos as atividades que cada grupo realizaria.

\subsection{Tecnologia: quadro e giz}

Observando a tecnologia quadro e giz, podemos notar (tabela 2) que, no grupo A, a grande maioria dos alunos tem dificuldade de representar o pentacloreto de fósforo $\left(\mathrm{PCl}_{5}\right)$, que apresenta uma

Tabela 2 - Tecnologia: Quadro e giz - Grupo A

\begin{tabular}{l|c|c|c|c|c|c|c|c|c|c|c}
\hline & $\mathbf{C S}_{2}$ & $\mathbf{F}_{2}$ & $\mathbf{H C N}$ & $\mathbf{C l B r}$ & $\mathbf{S O}_{2}$ & $\mathbf{C C l}_{4}$ & $\mathbf{S O}_{3}$ & $\mathbf{C O C l}_{2}$ & $\mathbf{S F}_{6}$ & $\mathbf{P C l}_{3}$ & $\mathbf{P C I}_{5}$ \\
\hline Não sei & $0,0 \%$ & $0,0 \%$ & $33,3 \%$ & $0,0 \%$ & $0,0 \%$ & $0,0 \%$ & $0,0 \%$ & $16,7 \%$ & $16,7 \%$ & $0,0 \%$ & $0,0 \%$ \\
\hline $\begin{array}{l}\text { Fiquei em } \\
\text { dúvida }\end{array}$ & $50,0 \%$ & $50,0 \%$ & $33,3 \%$ & $33,3 \%$ & $16,7 \%$ & $33,3 \%$ & $33,3 \%$ & $33,3 \%$ & $33,3 \%$ & $50,0 \%$ & $66,7 \%$ \\
\hline Não entendi & $0,0 \%$ & $0,0 \%$ & $0,0 \%$ & $0,0 \%$ & $0,0 \%$ & $0,0 \%$ & $0,0 \%$ & $0,0 \%$ & $0,0 \%$ & $0,0 \%$ & $0,0 \%$ \\
\hline Deu branco & $0,0 \%$ & $0,0 \%$ & $0,0 \%$ & $0,0 \%$ & $0,0 \%$ & $0,0 \%$ & $0,0 \%$ & $0,0 \%$ & $0,0 \%$ & $0,0 \%$ & $16,7 \%$ \\
\hline $\begin{array}{l}\text { Não deu } \\
\text { tempo }\end{array}$ & $0,0 \%$ & $0,0 \%$ & $0,0 \%$ & $0,0 \%$ & $0,0 \%$ & $0,0 \%$ & $0,0 \%$ & $0,0 \%$ & $0,0 \%$ & $0,0 \%$ & $0,0 \%$ \\
\hline Foi fácil & $50,0 \%$ & $50,0 \%$ & $33,3 \%$ & $66,7 \%$ & $83,3 \%$ & $66,7 \%$ & $66,7 \%$ & $50,0 \%$ & $50,0 \%$ & $50,0 \%$ & $16,7 \%$ \\
\hline Geometria & $\begin{array}{c}\text { Li- } \\
\text { near }\end{array}$ & $\begin{array}{c}\text { Li- } \\
\text { near }\end{array}$ & $\begin{array}{c}\text { Li- } \\
\text { near }\end{array}$ & $\begin{array}{c}\text { Li- } \\
\text { ne- } \\
\text { ar }\end{array}$ & $\begin{array}{c}\text { An- } \\
\text { gular }\end{array}$ & $\begin{array}{c}\text { Te- } \\
\text { traé- } \\
\text { drica }\end{array}$ & $\begin{array}{c}\text { Tri- } \\
\text { gonal }\end{array}$ & $\begin{array}{c}\text { Tri- } \\
\text { gonal }\end{array}$ & $\begin{array}{c}\text { Octa- } \\
\text { édrica }\end{array}$ & $\begin{array}{c}\text { Pira- } \\
\text { mi- } \\
\text { dal }\end{array}$ & $\begin{array}{c}\text { Bipirâ- } \\
\text { mide } \\
\text { trigonal }\end{array}$ \\
\hline
\end{tabular}


Tabela 3 - Tecnologia: Quadro e giz - Grupo D

\begin{tabular}{l|c|c|c|c|c|c|c|c|c|c|c}
\hline & $\mathbf{C S}_{2}$ & $\mathbf{F}_{2}$ & $\mathbf{H C N}$ & $\mathbf{C l B r}$ & $\mathbf{S O}_{2}$ & $\mathbf{C C l}_{4}$ & $\mathbf{S O}_{3}$ & $\mathbf{C O C l}_{2}$ & $\mathbf{S F}_{6}$ & $\mathbf{P C l}_{3}$ & $\mathbf{P C l}_{5}$ \\
\hline Não sei & $0,0 \%$ & $0,0 \%$ & $0,0 \%$ & $0,0 \%$ & $0,0 \%$ & $0,0 \%$ & $0,0 \%$ & $28,6 \%$ & $0,0 \%$ & $0,0 \%$ & $0,0 \%$ \\
\hline $\begin{array}{l}\text { Fiquei em } \\
\text { dúvida }\end{array}$ & $14,3 \%$ & $0,0 \%$ & $14,3 \%$ & $14,3 \%$ & $28,6 \%$ & $28,6 \%$ & $14,3 \%$ & $14,3 \%$ & $57,1 \%$ & $42,9 \%$ & $57,1 \%$ \\
\hline Não entendi & $0,0 \%$ & $0,0 \%$ & $0,0 \%$ & $0,0 \%$ & $0,0 \%$ & $0,0 \%$ & $0,0 \%$ & $0,0 \%$ & $0,0 \%$ & $0,0 \%$ & $0,0 \%$ \\
\hline Deu branco & $0,0 \%$ & $0,0 \%$ & $0,0 \%$ & $0,0 \%$ & $0,0 \%$ & $0,0 \%$ & $0,0 \%$ & $0,0 \%$ & $0,0 \%$ & $0,0 \%$ & $0,0 \%$ \\
\hline $\begin{array}{l}\text { Não deu } \\
\text { tempo }\end{array}$ & $0,0 \%$ & $0,0 \%$ & $0,0 \%$ & $0,0 \%$ & $0,0 \%$ & $0,0 \%$ & $0,0 \%$ & $0,0 \%$ & $0,0 \%$ & $0,0 \%$ & $0,0 \%$ \\
\hline Foi fácil & $85,7 \%$ & $100 \%$ & $85,7 \%$ & $85,7 \%$ & $71,4 \%$ & $71,4 \%$ & $85,7 \%$ & $57,1 \%$ & $42,9 \%$ & $57,1 \%$ & $42,9 \%$ \\
\hline & $\mathrm{Li}-$ & $\mathrm{Li}-$ & $\mathrm{Li}-$ & $\mathrm{Li}-$ & $\mathrm{Angu-}$ & $\begin{array}{c}\mathrm{Te} \text { - } \\
\text { traé- } \\
\text { Geometria } \\
\text { near }\end{array}$ & $\begin{array}{c}\text { Tri- } \\
\text { go- } \\
\text { near }\end{array}$ & $\begin{array}{c}\text { Tri- } \\
\text { go- } \\
\text { nal }\end{array}$ & $\begin{array}{c}\text { Octaé- } \\
\text { drica }\end{array}$ & $\begin{array}{c}\text { Pira- } \\
\text { midal }\end{array}$ & $\begin{array}{c}\text { Bipirâ- } \\
\text { mide } \\
\text { trigonal }\end{array}$ \\
\hline
\end{tabular}

geometria bipirâmide trigonal. Eles obtiveram também um menor percentual de acertos na representação. Isto ocorre pela complexidade da molécula que possui cinco orbitais no átomo central, cinco pares ligantes e nenhum par isolado, com ângulos de ligação de $180^{\circ}, 120^{\circ}$ e $90^{\circ}$. Representar o cianeto de hidrogênio (HCN) gerou algumas dúvidas entre os alunos, se seria uma geometria linear. Embora aparentemente seja uma representação considerada fácil, os alunos não constroem um modelo adequado. Talvez este fato possa ser justificado pela idéia de que a geometria linear acontece em toda molécula diatômica (que possui dois átomos) ou em toda molécula em que o átomo central possui no máximo duas nuvens eletrônicas em sua camada de valência. Alguns alunos não identificam as duas nuvens eletrônicas no átomo central em sua camada de valência.

Já no grupo D (tabela 3), observamos novamente a dificuldade em montar a molécula de
Pentacloreto de fósforo $\left(\mathrm{PCl}_{5}\right)$ de acordo com o modelo de VSEPR. Sintetizando, pode-se inferir que a combinação das tecnologias possibilita aos alunos uma compreensão melhor, pois os alunos deste grupo tiveram melhor desempenho após terem vivenciado uma situação de ensino com o software construindo um modelo mais adequado.

\subsection{Tecnologia: Bolas de isopor e varetas}

Os resultados encontrados no grupo B (tabela 4) sugerem que os compostos com estrutura linear e bipirâmide pentagonal são aqueles que apresentam obstáculos inerentes ao aprendizado. Tais obstáculos podem estar associados a aspectos conceituais ou aspectos que correspondem à imagem mental elaborada pelos alunos. Na formação da imagem mental ou modelo construído pelo aluno a representação gráfica ou concreta (varetas) desempenha papel fundamental. Tal representação tem suas

Tabela 4 - Tecnologia: Bolas de isopor e varetas - grupo B

\begin{tabular}{l|c|c|c|c|c|c|c|c|c|c|c}
\hline & $\mathbf{C S}_{2}$ & $\mathbf{F}_{2}$ & $\mathbf{H C N}$ & $\mathbf{C l B r}$ & $\mathbf{S O}_{2}$ & $\mathbf{C C l}_{4}$ & $\mathbf{S O}_{3}$ & $\mathbf{C O C l}_{2}$ & $\mathbf{S F}_{6}$ & $\mathbf{P C l}_{3}$ & $\mathbf{P C l}_{5}$ \\
\hline Não sei & $0,0 \%$ & $0,0 \%$ & $0,0 \%$ & $0,0 \%$ & $0,0 \%$ & $0,0 \%$ & $0,0 \%$ & $12,5 \%$ & $12,5 \%$ & $0,0 \%$ & $0,0 \%$ \\
\hline $\begin{array}{l}\text { Fiquei em } \\
\text { dúvida }\end{array}$ & $12,5 \%$ & $0,0 \%$ & $50,0 \%$ & $0,0 \%$ & $50,0 \%$ & $12,5 \%$ & $25,0 \%$ & $12,5 \%$ & $25,0 \%$ & $25,0 \%$ & $25,0 \%$ \\
\hline Não entendi & $0,0 \%$ & $0,0 \%$ & $0,0 \%$ & $0,0 \%$ & $0,0 \%$ & $0,0 \%$ & $12,5 \%$ & $25,0 \%$ & $0,0 \%$ & $12,5 \%$ & $12,5 \%$ \\
\hline Deu branco & $0,0 \%$ & $0,0 \%$ & $0,0 \%$ & $0,0 \%$ & $0,0 \%$ & $0,0 \%$ & $0,0 \%$ & $0,0 \%$ & $0,0 \%$ & $0,0 \%$ & $0,0 \%$ \\
\hline $\begin{array}{l}\text { Não deu } \\
\text { tempo }\end{array}$ & $0,0 \%$ & $0,0 \%$ & $12,5 \%$ & $0,0 \%$ & $12,5 \%$ & $0,0 \%$ & $12,5 \%$ & $25,0 \%$ & $0,0 \%$ & $0,0 \%$ & $37,5 \%$ \\
\hline Foi fácil & $87,5 \%$ & $100 \%$ & $37,5 \%$ & $100 \%$ & $37,5 \%$ & $87,5 \%$ & $50,0 \%$ & $25,0 \%$ & $62,5 \%$ & $62,5 \%$ & $25,0 \%$ \\
\hline & Li- & $\begin{array}{c}\text { Li- } \\
\text { Gear }\end{array}$ & $\begin{array}{c}\text { Li- } \\
\text { near }\end{array}$ & $\begin{array}{c}\text { Li- } \\
\text { near }\end{array}$ & $\begin{array}{c}\text { Angu- } \\
\text { lar }\end{array}$ & $\begin{array}{c}\text { Té- } \\
\text { traé- } \\
\text { drica }\end{array}$ & $\begin{array}{c}\text { Tri- } \\
\text { gonal }\end{array}$ & $\begin{array}{c}\text { Tri- } \\
\text { gonal }\end{array}$ & $\begin{array}{c}\text { Octaé- } \\
\text { drica }\end{array}$ & $\begin{array}{c}\text { Pira- } \\
\text { midal }\end{array}$ & $\begin{array}{c}\text { Bipirâ- } \\
\text { mide } \\
\text { trigo- } \\
\text { nal }\end{array}$ \\
\hline
\end{tabular}


Tabela 5 - Tecnologia: Bolas de isopor e varetas - grupo C

\begin{tabular}{l|c|c|c|c|c|c|c|c|c|c|c}
\hline & $\mathbf{C S}_{2}$ & $\mathbf{F}_{2}$ & $\mathbf{H C N}$ & $\mathbf{C l B r}$ & $\mathbf{S O}_{2}$ & $\mathbf{C C l}_{4}$ & $\mathbf{S O}_{3}$ & $\mathbf{C O C l}_{2}$ & $\mathbf{S F}_{6}$ & $\mathbf{P C l}_{3}$ & $\mathbf{P C I}_{5}$ \\
\hline Não sei & $14,3 \%$ & $0,0 \%$ & $0,0 \%$ & $28,6 \%$ & $14,3 \%$ & $0,0 \%$ & $0,0 \%$ & $14,3 \%$ & $14,3 \%$ & $14,3 \%$ & $28,6 \%$ \\
\hline $\begin{array}{l}\text { Fiquei em } \\
\text { dúvida }\end{array}$ & $28,6 \%$ & $0,0 \%$ & $14,3 \%$ & $14,3 \%$ & $28,6 \%$ & $42,9 \%$ & $28,6 \%$ & $28,6 \%$ & $57,1 \%$ & $28,6 \%$ & $71,4 \%$ \\
\hline $\begin{array}{l}\text { Não en- } \\
\text { tendi }\end{array}$ & $0,0 \%$ & $0,0 \%$ & $0,0 \%$ & $0,0 \%$ & $0,0 \%$ & $42,9 \%$ & $14,3 \%$ & $0,0 \%$ & $0,0 \%$ & $0,0 \%$ & $0,0 \%$ \\
\hline Deu branco & $0,0 \%$ & $14,3 \%$ & $0,0 \%$ & $0,0 \%$ & $0,0 \%$ & $0,0 \%$ & $0,0 \%$ & $0,0 \%$ & $0,0 \%$ & $0,0 \%$ & $0,0 \%$ \\
\hline $\begin{array}{l}\text { Não deu } \\
\text { tempo }\end{array}$ & $0,0 \%$ & $0,0 \%$ & $0,0 \%$ & $0,0 \%$ & $0,0 \%$ & $0,0 \%$ & $0,0 \%$ & $0,0 \%$ & $0,0 \%$ & $0,0 \%$ & $0,0 \%$ \\
\hline Foi fácil & $57,1 \%$ & $85,7 \%$ & $85,7 \%$ & $57,1 \%$ & $57,1 \%$ & $14,3 \%$ & $57,1 \%$ & $57,1 \%$ & $28,6 \%$ & $57,1 \%$ & $0,0 \%$ \\
\hline & Li- & Li- & Li- & Li- & An- & $\begin{array}{c}\text { Te- } \\
\text { neara- } \\
\text { near }\end{array}$ & $\begin{array}{c}\text { Tri- } \\
\text { gonal }\end{array}$ & $\begin{array}{c}\text { Trigo- } \\
\text { nal }\end{array}$ & $\begin{array}{c}\text { Octa- } \\
\text { édrica }\end{array}$ & $\begin{array}{c}\text { Pira- } \\
\text { midal }\end{array}$ & $\begin{array}{c}\text { Bipide } \\
\text { trigo- } \\
\text { nal }\end{array}$ \\
\hline
\end{tabular}

especificidades que, ao ser modelada, pode guardar características que não pertencem a estrutura, por desconsiderar as propriedades e a correta localização dos átomos.

No grupo C, a representação dos compostos pentacloreto de fósforo $\left(\mathrm{PCl}_{5}\right)$ e tetracloreto de carbono $\left(\mathrm{CCl}_{4}\right)$ pelos alunos apresentam em sua construção aspectos que não são bem claros, com desenhos particulares. Embora o tetracloreto de carbono pareça ser uma estrutura fácil de se prever, a geometria teve baixo percentual de acertos. Talvez a origem do problema possa estar nas próprias demonstrações do professor/pesquisador que utilizou argumentos ordenados e prontos, com um tratamento estereotipado que leva os alunos a não reconhecerem qual a estrutura adequada de moléculas que apresentam o mesmo tipo de geometria molecular. Já o composto cianeto de hidrogênio (HCN), que apresentou dificuldades nos outros grupos, tem aqui o seu maior valor percentual de facilidade em toda a pesquisa. Os resultados obtidos pelo grupo estão na tabela 5.

\subsection{Tecnologia: software chemsketch ${ }^{\circledR}$}

Para o grupo C, considerando as dificuldades na utilização do software, é possível revelar que $57,1 \%$ apresentaram-nas algumas vezes, $28,6 \%$ quase sempre e 14,3\% não tiveram nenhuma dificuldade. $42,9 \%$ dos pesquisados sempre executaram as tarefas propostas na ordem solicitada, 28,6\% quase sempre e $28,6 \%$ também algumas vezes.
$71,4 \%$ dos pesquisados solicitaram, algumas vezes, ajuda ao professor para executar as tarefas propostas, $14,3 \%$, sempre e $14,3 \%$, quase sempre. $71,4 \%$ dos pesquisados quase sempre analisaram corretamente os ângulos formados, observando os vetores criados, enquanto $14,3 \%$ sempre o faziam e $14,3 \%$, algumas vezes.

$85,7 \%$ concordam que esse tipo de software deveria ser utilizado mais vezes nas aulas e 14,3\% discordam.

Com relação ao grupo C (tabela 6), os valores significativos nos compostos com geometria linear podem caracterizar que ocorreu aprendizagem entre os alunos. Já não é possível dizer dos compostos pentacloreto de fósforo $\left(\mathrm{PCl}_{5}\right)$ e fosfogênio $\left(\mathrm{COCl}_{2}\right)$, que tiveram baixíssimos valores. O pentacloreto de fósforo, como já dito, apresenta uma maior dificuldade para a sua montagem, em função do tipo de geometria que apresenta.

Os alunos do grupo D que interagiram com o software apresentaram dificuldades na execução do software, solicitando a presença do professor constantemente. Também foi observado que alguns alunos não iniciaram imediatamente a execução do software e a construção das estruturas.

Considerando as dificuldades na utilização do software, 57,1\% apresentaram-nas algumas vezes, $28,6 \%$, quase sempre e $14,3 \%$ não tiveram nenhuma dificuldade. 42,9\% dos pesquisados sempre executaram as tarefas propostas na ordem solicitada, 28,6\%, quase sempre e $28,6 \%$, também, algumas vezes. 
Tabela 6 - Tecnologia: Software ChemSketch ${ }^{\circledR}$ - grupo C

\begin{tabular}{l|c|c|c|c|c|c|c|c|c|c|c}
\hline & $\mathbf{C S}_{2}$ & $\mathbf{F}_{2}$ & $\mathbf{H C N}$ & $\mathbf{C l B r}$ & $\mathbf{S O}_{2}$ & $\mathbf{C C l}_{4}$ & $\mathbf{S O}_{3}$ & $\mathbf{C O C l}_{2}$ & $\mathbf{S F}_{6}$ & $\mathbf{P C l}_{3}$ & $\mathbf{P C l}_{5}$ \\
\hline Não sei & $14,3 \%$ & $0,0 \%$ & $14,3 \%$ & $0,0 \%$ & $0,0 \%$ & $0,0 \%$ & $0,0 \%$ & $0,0 \%$ & $28,6 \%$ & $14,3 \%$ & $14,3 \%$ \\
\hline $\begin{array}{l}\text { Fiquei em } \\
\text { dúvida }\end{array}$ & $14,3 \%$ & $14,3 \%$ & $14,3 \%$ & $0,0 \%$ & $42,9 \%$ & $42,9 \%$ & $42,9 \%$ & $57,1 \%$ & $14,3 \%$ & $28,6 \%$ & $57,1 \%$ \\
\hline Não entendi & $0,0 \%$ & $14,3 \%$ & $0,0 \%$ & $0,0 \%$ & $0,0 \%$ & $0,0 \%$ & $28,5 \%$ & $14,3 \%$ & $14,3 \%$ & $0,0 \%$ & $0,0 \%$ \\
\hline Deu branco & $0,0 \%$ & $0,0 \%$ & $0,0 \%$ & $0,0 \%$ & $0,0 \%$ & $0,0 \%$ & $0,0 \%$ & $0,0 \%$ & $0,0 \%$ & $0,0 \%$ & $0,0 \%$ \\
\hline $\begin{array}{l}\text { Não deu } \\
\text { tempo }\end{array}$ & $0,0 \%$ & $0,0 \%$ & $0,0 \%$ & $0,0 \%$ & $14,3 \%$ & $0,0 \%$ & $0,0 \%$ & $14,3 \%$ & $0,0 \%$ & $0,0 \%$ & $14,3 \%$ \\
\hline Foi fácil & $71,4 \%$ & $71,4 \%$ & $71,4 \%$ & $100 \%$ & $42,9 \%$ & $57,1 \%$ & $28,6 \%$ & $14,3 \%$ & $42,9 \%$ & $57,1 \%$ & $14,3 \%$ \\
\hline Geometria & $\begin{array}{c}\text { Li- } \\
\text { near }\end{array}$ & $\begin{array}{c}\text { Li- } \\
\text { near }\end{array}$ & $\begin{array}{c}\text { Li- } \\
\text { near }\end{array}$ & $\begin{array}{c}\text { Li- } \\
\text { near }\end{array}$ & $\begin{array}{c}\text { An- } \\
\text { gular }\end{array}$ & $\begin{array}{c}\text { Traé- } \\
\text { drica }\end{array}$ & $\begin{array}{c}\text { Tri- } \\
\text { gonal }\end{array}$ & $\begin{array}{c}\text { Tri- } \\
\text { gonal }\end{array}$ & $\begin{array}{c}\text { Octa- } \\
\text { édri- } \\
\text { ca }\end{array}$ & $\begin{array}{c}\text { Pira- } \\
\text { midal }\end{array}$ & $\begin{array}{c}\text { Bipirâ- } \\
\text { mide } \\
\text { trigonal }\end{array}$ \\
\hline
\end{tabular}

85,7\% dos pesquisados solicitaram algumas vezes ajuda ao professor para executar as tarefas propostas e $14,3 \%$, nunca. $14,3 \%$ dos pesquisados sempre analisaram corretamente os ângulos formados, observando os vetores criados; $57,1 \%$, algumas vezes e $28,6 \%$ quase sempre.

$85,7 \%$ concordam que este tipo de software deveria ser utilizado mais vezes nas aulas, e 14,3\% discordam. Os resultados obtidos pelo grupo C encontram-se na tabela 7 .

No grupo $\mathrm{A}$, no tocante às dificuldades na utilização do software, 71,4\% apresentaram-nas algumas vezes, $14,3 \%$, sempre e $14,3 \%$ não tiveram nenhuma dificuldade. $57,1 \%$ dos pesquisados sempre executaram as tarefas propostas na ordem solicitada; $28,6 \%$, quase sempre e $14,3 \%$, algumas vezes.

Segundo a avaliação dos próprios alunos, 42,9\% sempre analisaram corretamente os ângulos formados, observando os vetores criados; $14,3 \%$, quase sempre; 28,5\%, algumas vezes e 14,3\% nunca observavam esses ângulos. Os resultados obtidos pelo grupo A encontram-se na tabela 8 .

No grupo $\mathrm{B}$, no tocante às dificuldades na utilização do software, $100 \%$ apresentaram-nas algumas vezes, $62,5 \%$ dos pesquisados sempre executaram as tarefas propostas na ordem solicitada, enquanto $37,5 \%$, quase sempre. $12,5 \%$ dos pesquisados sempre analisaram corretamente os ângulos formados, observando os vetores criados, enquanto $25 \%$, quase sempre, $50 \%$, algumas vezes e $12,5 \%$, nunca.

$50 \%$ dos pesquisados concordam totalmente que esse tipo de software deveria ser utilizado mais vezes nas aulas, enquanto os outros $50 \%$ concordam parcialmente.Os resultados obtidos encontram-se na tabela 9.

O grupo A realizou a atividade após uma aula expositiva com a tecnologia de quadro e giz e repetiu a mesma atividade com computador no

Tabela 7 - Tecnologia: Software ChemSketch ${ }^{\circledR}$ - grupo D

\begin{tabular}{l|c|c|c|c|c|c|c|c|c|c|c}
\hline & $\mathbf{C S}_{2}$ & $\mathbf{F}_{2}$ & $\mathbf{H C N}$ & $\mathbf{C l B r}$ & $\mathbf{S O}_{2}$ & $\mathbf{C C l}_{4}$ & $\mathbf{S O}_{3}$ & $\mathbf{C O C l}_{2}$ & $\mathbf{S F}_{6}$ & $\mathbf{P C l}_{3}$ & $\mathbf{P C l}_{5}$ \\
\hline Não sei & $0,0 \%$ & $14,3 \%$ & $14,3 \%$ & $14,3 \%$ & $0,0 \%$ & $14,3 \%$ & $0,0 \%$ & $28,6 \%$ & $0,0 \%$ & $14,3 \%$ & $\mathrm{PCl} 5$ \\
\hline $\begin{array}{l}\text { Fiquei em } \\
\text { dúvida }\end{array}$ & $57,1 \%$ & $14,3 \%$ & $42,9 \%$ & $0,0 \%$ & $28,6 \%$ & $14,3 \%$ & $42,9 \%$ & $28,6 \%$ & $71,4 \%$ & $28,6 \%$ & $42,9 \%$ \\
\hline Não entendi & $0,0 \%$ & $0,0 \%$ & $0,0 \%$ & $0,0 \%$ & $28,6 \%$ & $0,0 \%$ & $14,3 \%$ & $0,0 \%$ & $0,0 \%$ & $0,0 \%$ & $0,0 \%$ \\
\hline Deu branco & $0,0 \%$ & $0,0 \%$ & $0,0 \%$ & $0,0 \%$ & $0,0 \%$ & $0,0 \%$ & $0,0 \%$ & $0,0 \%$ & $0,0 \%$ & $0,0 \%$ & $0,0 \%$ \\
\hline $\begin{array}{l}\text { Não deu } \\
\text { tempo }\end{array}$ & $0,0 \%$ & $0,0 \%$ & $0,0 \%$ & $0,0 \%$ & $0,0 \%$ & $0,0 \%$ & $0,0 \%$ & $0,0 \%$ & $0,0 \%$ & $0,0 \%$ & $0,0 \%$ \\
\hline Foi fácil & $42,9 \%$ & $71,4 \%$ & $42,9 \%$ & $85,7 \%$ & $42,9 \%$ & $71,4 \%$ & $42,9 \%$ & $42,9 \%$ & $28,6 \%$ & $57,1 \%$ & $42,9 \%$ \\
\hline Geometria & $\begin{array}{c}\text { Li- } \\
\text { near }\end{array}$ & $\begin{array}{c}\text { Li- } \\
\text { near }\end{array}$ & $\begin{array}{c}\text { Li- } \\
\text { near }\end{array}$ & $\begin{array}{c}\mathrm{Li}- \\
\text { near }\end{array}$ & $\begin{array}{c}\text { An- } \\
\text { gular }\end{array}$ & $\begin{array}{c}\text { Tédrica } \\
\text { édrica }\end{array}$ & $\begin{array}{c}\text { Tri- } \\
\text { gonal }\end{array}$ & $\begin{array}{c}\text { Tri- } \\
\text { gonal }\end{array}$ & $\begin{array}{c}\text { Octa- } \\
\text { édri- } \\
\text { ca }\end{array}$ & $\begin{array}{c}\text { Pira- } \\
\text { midal }\end{array}$ & $\begin{array}{c}\text { Bipirâ- } \\
\text { mide } \\
\text { trigonal }\end{array}$ \\
\hline
\end{tabular}


Tabela 8 - Atividade com o software ChemSketch - grupo A

\begin{tabular}{l|c|c|c|c|c|c|c|c|c|c|c}
\hline & $\mathbf{C S}_{2}$ & $\mathbf{F}_{2}$ & $\mathbf{H C N}$ & $\mathbf{C l B r}$ & $\mathbf{S O}_{2}$ & $\mathbf{C C l}_{4}$ & $\mathbf{S O}_{3}$ & $\mathbf{C O C l}_{2}$ & $\mathbf{S F}_{6}$ & $\mathbf{P C l}_{3}$ & $\mathbf{P C l}_{5}$ \\
\hline Não sei & $0,0 \%$ & $14,3 \%$ & $14,3 \%$ & $14,3 \%$ & $0,0 \%$ & $0,0 \%$ & $0,0 \%$ & $28,6 \%$ & $14,3 \%$ & $14,3 \%$ & $28,6 \%$ \\
\hline $\begin{array}{l}\text { Fiquei em } \\
\text { dúvida }\end{array}$ & $57,1 \%$ & $28,6 \%$ & $57,1 \%$ & $42,9 \%$ & $42,9 \%$ & $71,4 \%$ & $57,1 \%$ & $57,1 \%$ & $42,9 \%$ & $57,1 \%$ & $42,9 \%$ \\
\hline Não entendi & $0,0 \%$ & $0,0 \%$ & $14,3 \%$ & $0,0 \%$ & $0,0 \%$ & $0,0 \%$ & $0,0 \%$ & $0,0 \%$ & $0,0 \%$ & $0,0 \%$ & $0,0 \%$ \\
\hline Deu branco & $0,0 \%$ & $0,0 \%$ & $0,0 \%$ & $0,0 \%$ & $0,0 \%$ & $0,0 \%$ & $0,0 \%$ & $0,0 \%$ & $0,0 \%$ & $0,0 \%$ & $0,0 \%$ \\
\hline $\begin{array}{l}\text { Não deu } \\
\text { tempo }\end{array}$ & $0,0 \%$ & $0,0 \%$ & $0,0 \%$ & $0,0 \%$ & $0,0 \%$ & $0,0 \%$ & $0,0 \%$ & $0,0 \%$ & $0,0 \%$ & $0,0 \%$ & $0,0 \%$ \\
\hline Foi fácil & $42,9 \%$ & $57,1 \%$ & $14,3 \%$ & $42,9 \%$ & $57,1 \%$ & $28,6 \%$ & $42,9 \%$ & $14,3 \%$ & $42,9 \%$ & $28,6 \%$ & $28,6 \%$ \\
\hline Geometria & $\begin{array}{c}\text { Li- } \\
\text { near }\end{array}$ & $\begin{array}{c}\text { Li- } \\
\text { near }\end{array}$ & $\begin{array}{c}\text { Li- } \\
\text { near }\end{array}$ & $\begin{array}{c}\text { Li- } \\
\text { near }\end{array}$ & $\begin{array}{c}\text { An- } \\
\text { gular }\end{array}$ & $\begin{array}{c}\text { Tédra- } \\
\text { édrica }\end{array}$ & $\begin{array}{c}\text { Tri- } \\
\text { gonal }\end{array}$ & $\begin{array}{c}\text { Tri- } \\
\text { gonal }\end{array}$ & $\begin{array}{c}\text { Octa- } \\
\text { édri- } \\
\text { ca }\end{array}$ & $\begin{array}{c}\text { Pira- } \\
\text { midal }\end{array}$ & $\begin{array}{c}\text { Bipirâ- } \\
\text { mide } \\
\text { trigonal }\end{array}$ \\
\hline
\end{tabular}

laboratório de tecnologia educacional. O grupo D fez o inverso: passou primeiramente pelo laboratório de tecnologia educacional e depois foi para a aula com quadro e giz. Os resultados trazem à luz algumas observações que serão importantes para o resultado do trabalho.

O grupo A apresentou menor percentual de dificuldades com relação à utilização do software. Isso é justificado porque eles tinham uma visão mais ampliada do tema em estudo já que haviam realizado a tarefa com o auxílio de uma outra tecnologia.

O grupo D apresenta um maior percentual de respostas positivas quando é indagado se a utilização do software pode auxiliar no entendimento de geometria molecular. É importante ressaltar que estavam trabalhando com o software primeiro e não tinham uma visão tão ampla com relação ao questionamento.

No grupo A, a maioria dos alunos respondeu que a utilização do software favorecia uma melhor compreensão e execução das tarefas propostas. Isso reforça a idéia de que o software deve ser utilizado como uma ferramenta de apoio inserido em uma proposta de ensino e não simplesmente como um fato isolado.

Os resultados da investigação sugerem que a utilização do software ChemSketch ${ }^{\circledR}$ por parte dos alunos é bem aceita. Ainda assim, verifica-se que algumas dificuldades ocorreram, como por exemplo, os alunos tentavam abrir novamente a tabela periódica sendo que ela já estava já aberta, demonstrando uma desorientação em relação à aplicação do software e também da atividade que estava sendo desenvolvida

Na investigação também foram observadas dificuldades na compreensão dos conceitos químicos, como por exemplo, o aluno tentava ligar o elemento flúor com outro átomo de flúor, que estava ligado ao enxofre na tentativa de montar o $\mathrm{SF}_{6}$, demonstrando

Tabela 9-Atividade com o software ChemSketch ${ }^{\circledR}$ - grupo B

\begin{tabular}{l|c|c|c|c|c|c|c|c|c|c|c}
\hline & $\mathbf{C S}_{2}$ & $\mathbf{F}_{2}$ & $\mathbf{H C N}$ & $\mathbf{C I B r}$ & $\mathbf{S O}_{2}$ & $\mathbf{C C l}_{4}$ & $\mathbf{S O}_{3}$ & $\mathbf{C O C l}_{2}$ & $\mathbf{S F}_{6}$ & $\mathbf{P C l}_{3}$ & $\mathbf{P C l}_{5}$ \\
\hline Não sei & $12,5 \%$ & $0,0 \%$ & $25,0 \%$ & $0,0 \%$ & $0,0 \%$ & $0,0 \%$ & $0,0 \%$ & $12,5 \%$ & $0,0 \%$ & $0,0 \%$ & $0,0 \%$ \\
\hline $\begin{array}{l}\text { Fiquei em } \\
\text { dúvida }\end{array}$ & $12,5 \%$ & $0,0 \%$ & $37,5 \%$ & $12,5 \%$ & $12,5 \%$ & $25,0 \%$ & $50,0 \%$ & $50,0 \%$ & $25,0 \%$ & $37,5 \%$ & $50,0 \%$ \\
\hline Não entendi & $0,0 \%$ & $0,0 \%$ & $12,5 \%$ & $0,0 \%$ & $0,0 \%$ & $0,0 \%$ & $12,5 \%$ & $12,5 \%$ & $0,0 \%$ & $0,0 \%$ & $12,5 \%$ \\
\hline Deu branco & $0,0 \%$ & $0,0 \%$ & $0,0 \%$ & $0,0 \%$ & $0,0 \%$ & $0,0 \%$ & $12,5 \%$ & $0,0 \%$ & $12,5 \%$ & $12,5 \%$ & $0,0 \%$ \\
\hline $\begin{array}{l}\text { Não deu } \\
\text { tempo }\end{array}$ & $0,0 \%$ & $0,0 \%$ & $0,0 \%$ & $0,0 \%$ & $0,0 \%$ & $0,0 \%$ & $0,0 \%$ & $0,0 \%$ & $0,0 \%$ & $0,0 \%$ & $0,0 \%$ \\
\hline Foi fácil & $75,0 \%$ & $100 \%$ & $25,0 \%$ & $87,5 \%$ & $87,5 \%$ & $75,0 \%$ & $25,0 \%$ & $25,0 \%$ & $62,5 \%$ & $50,0 \%$ & $37,5 \%$ \\
\hline Geometria & $\begin{array}{c}\mathrm{Li}- \\
\text { near }\end{array}$ & $\begin{array}{c}\text { Li- } \\
\text { near }\end{array}$ & $\begin{array}{c}\text { Li- } \\
\text { near }\end{array}$ & $\begin{array}{c}\text { Li- } \\
\text { near }\end{array}$ & $\begin{array}{c}\text { An- } \\
\text { gular }\end{array}$ & $\begin{array}{c}\text { Te- } \\
\text { traé- } \\
\text { drica }\end{array}$ & $\begin{array}{c}\text { Tri- } \\
\text { gonal }\end{array}$ & $\begin{array}{c}\text { Tri- } \\
\text { gonal }\end{array}$ & $\begin{array}{c}\text { Octa- } \\
\text { édrica }\end{array}$ & $\begin{array}{c}\text { Pira- } \\
\text { midal }\end{array}$ & $\begin{array}{c}\text { Bipirâ- } \\
\text { mide } \\
\text { trigonal }\end{array}$ \\
\hline
\end{tabular}


assim total desconhecimento com relação às ligações que o flúor pode efetivamente realizar. Também foi notada a dificuldade em interligar os elementos para que possam ser efetuadas as ligações entre eles. Elementos também apresentavam valências incompatíveis com as ligações reais.

Os resultados das análises sugerem que a utilização do software SchemSketch ${ }^{\circledR}$ pode propiciar a visualização tridimensional das moléculas. A facilidade de utilização pelo alunos, como por exemplo o acesso à tabela periódica durante a execução da pesquisa. Pode também apresentar moléculas muito bem montadas com angulações perfeitas em relação ao modelo VSEPR.

\section{Conclusão e recomendações}

As aprendizagens que envolvem o estudo do tópico Geometria Molecular são particularmente complexas, pois os alunos têm dificuldades na previsão das formas das moléculas.(BIRK; KURTZ; 1999; FURIO; 1996; LOBO; 1998). Para minimizar esta situação são criados dispositivos simples para reproduzir as principais geometrias e, assim, facilitar as aprendizagens dos alunos. Nas abordagens didático-pedagógicas usuais tem prevalecido a utilização de desenhos no quadro de giz, cartazes, bolas de isopor e varetas, bexigas de ar, massas de modelar, modelos comerciais. Raramente os professores lançam mão dos softwares.

Com a preocupação de favorecer uma aprendizagem mais significativa e efetiva para os alunos no tópico do conteúdo sobre Geometria Molecular, buscou-se utilizar e integrar as tecnologias na perspectiva de proporcionar várias formas de captar e mostrar o mesmo objeto, representando-o sob ângulos e meios diferentes: pelos movimentos, mudanças de cenários, integração de formas mais tradicionais de ensino com tecnologias mais avançadas, o dedutivo e o indutivo, o espaço e o tempo, o concreto e o abstrato. Assim, foram utilizadas diferentes tecnologias para representação das moléculas - algumas mais abstratas (desenhos), outras mais concretas (varetas, bolas de isopor...), computacionais (3D) -, mas, todas elas, combinadas, integradas, possi- bilitaram maior compreensão do conteúdo.

A integração das diferentes tecnologias possibilitou aos alunos o contato com outras linguagens e códigos, motivando-os nas aprendizagens, pois, embora o conteúdo explorado nas situações de ensino fosse o mesmo, este adquiriu uma outra dimensão, visto que permitiu diferentes percursos de aprendizagem.

A utilização do software ChemSketch ${ }^{\circledR}$ indicia que as aprendizagens não foram significativas em relação às outras situações de ensino que contemplaram outras tecnologias. Tal resultado pode reduzir o entusiasmo de quem acredita que as tecnologias podem curar todos os males da educação. Nesse sentido, é necessário refletir sobre como usar essa tecnologia. É claro que esse ponto não significa que a utilização do software seja o problema. Todavia, há uma série de intervenientes que podem influenciar as aprendizagens dos alunos. Um dos intervenientes decorre da visão fragmentada sobre a atividade, perpassada pela idéia de que o aluno deve seguir uma seqüência lógica do tipo "faça isto deste modo”, “faça aquilo deste modo”, “Clike aqui”. Nesse sentido, ao se defrontar com uma dificuldade na interface, o aluno acaba se perdendo. Outro interveniente é a própria concepção do professor que esquece ser o software apenas uma ferramenta capaz de permitir uma aprendizagem mais significativa. Esquece que a ferramenta "per si" não promove a aprendizagem e que é fundamental a presença do professor atuando de forma ativa e criativa nesse processo. Assim, é possível propor abordagens de aprendizagem construtivistas, centradas no aluno e colaborativas. Todavia, não há nada inerente ao computador que conduza a isso. Nesse caso, ele pode também ser utilizado para dar suporte a cursos e programas tradicionais, centrados no professor e baseados na transmissão de conhecimentos. Tudo depende da concepção de ensino e aprendizagem daquele que determina como será instaurado tal processo.

Desse modo, numa perspectiva de construção dos conhecimentos, o professor não pode limitarse a uma comunicação unilateral, caracterizada pela transmissão de conteúdos. Ele passa a ser um orientador, que apresenta modelos, que facilita as 
interações, faz mediações, dá explicações, (re)direciona o foco e oferece opções a seus alunos.

A utilização do software de modo integrado favorece uma maior compreensão do aluno na proposição de formas representacionais das moléculas.

Ao investigar a utilização de diferentes tecnologias no ensino de Geometria Molecular, não se teve a pretensão de prescrever como deveriam ser as aulas de Química sobre o tema em estudo a fim de se efetivar as aprendizagens. Nessa perspectiva, não se quer especificar "receitas" e/ou abordagens de ensino que possam ser consideradas mais efetivas nas aprendizagens dos alunos, por serem mais envolventes ou mais motivadoras. Pois isso não significa garantia de sucesso nas aprendizagens. Ainda, uma postura assim simplificaria o conhecimento e a prática de ensino de Química. Nesses termos, pretende-se abrir um leque de discussões que considere as possibilidades presentes nas práticas de ensino de Química, atentando para a necessidade da criação de ambientes de aprendizagem, nos quais os alunos possam interagir com uma variedade de tecnologias combinadas, capazes, então, de possibilitar a construção do conhecimento.

Desse modo, o trabalho não conduz a uma conclusão, mas abre perspectivas, como ponto de partida, para futuras pesquisas que, utilizando os pressupostos aqui discutidos, possam apontar um campo bastante amplo, para o tratamento investigativo sobre os processos de ensino e aprendizagem mediados pelo computador e a integração de diferentes tecnologias, que merecem novas investigações.

\section{REFERÊNCIAS}

ANJOS, E. I. ; GIORDAN, M. . Visualização molecular como estratégia para ensinar química orgânica. In: XI Encontro Nacional de Ensino de Química, 2002, Recife. Livro de resumos, 2002. v. 1. p. 167-167.

ANJOS, E. I. Modelos mentais e visualização molecular: uma estratégia para ensinar química orgânica. 2004. 120p. Dissertação (Mestrado). Instituto de Física, Instituto de Química, Faculdade de Educação, Universidade de São Paulo, 2004.

BARNEA, N. ; DORI, Y. J. High school chemistry students' performance and gender differences in a computerized mo- lecular modeling learning environment. Journal of Science Education and Technology. v. 8, n. 4, p. 257-271, 1999.

. Computerized molecular modeling the new technology for enhancing model perception among chemistry educators and learners. Chemistry Education: Research and Practice in Europe. v. 1, n. 1, p. 109-120, 2000. <http:// www.uoi.gr/conf_sem/cerapie/pdf/16barneaf.pdf $>$ Acesso em: 20 set 2006.

BIRK, J. P., KURTZ, M. J. Effect of Experience on Retention and Elimination of Misconceptions about Molecular Structure and Bonding. Journal of Chemical Education. n. 76, p. 124-128, 1999.

BONILLA, M. H. S. Escola Aprendente: para além da sociedade da informação. Rio de Janeiro: Quartet, 2005. 224p.

BROOK, D. W. Science education notes: The teaching of organic chemistry in school - Can we learn from the Kenyan experience. School Science Review, v. 69, n. 248, p. 575 578, 1988.

CHANG, C.-Y. Comparing the Impacts of a Problem-based Computer-Assisted Instruction and the Direct-Interactive Teaching Method on Student Science Achievement. Journal of Science Education and Technology, v.10, n.2, p.147-153, 2001.

COPOLO, C. F.; HOUNSHELL, P. B. Using three-dimensional models to teach molecular structures in high school chemistry. Journal of Science Education and Technology. v. 4. n. 4, p. 295-305, 1995.

DORI, Y. J. Cooperative development of organic chemistry computer assisted instruction by experts, teachers and students. Journal of Science Education and Technology, v. 4, n. 2, p. 163-170, 1995.

DORI, Y. J.; HAMEIRI, M. The “Mole Environment” study ware: Applying multidimensional analysis to quantitative chemistry problems. International Journal of Science Education, v. 20, n. 3, p. 317-333, 1998.

EICHLER, M. L.; DEL PINO, J. C. Ambientes virtuais de aprendizagem. Porto Alegre: Editora UFRGS, 2006. 175p.

FURIÓ, C.; CALATAYUD, M. L. Difficulties with the geometry and polarity of molecules. Beyond misconceptions. Journal of Chemical Education, v. 73, p. 36-41, 1996.

GABEL, D.; Bunce, D.M. Research on problem solving: Chemistry. In D. Gabel (Ed.). Handbook of Research on Science Teaching and Learning. p. 301-326. Macmillan, New York, 1994.

GASTEIGER, J.; ENGEL, T. Chemoinformatics: a textbook. Germany: Wiley-VCH. 2003. 680p.

GILBERT, J. K; BOULTER, C; RUTHERFORD, M. Explanations with Models in Science Education. In: GILBERT, J. K; BOULTER, C. Developing models in science education. 
First Edition. Netherlands: Kluwer Academic Publishers. p. 193-208, 2000.

GILBERT, J. K.; BOULTER, C. J. Stretching models too far. Paper presented at the annual meeting of the American Educational Research Association, San Francisco. Apr. 1995

GILLESPIE, R. J., ROBINSON, E. A. Electron Domains and the VSEPR Model of Molecular Geometry. Angewandte Chemie International Edition in English. n. 33, p. 495-514, 1996.

GILLESPIE, R. J. Teaching Molecular Geometry with the VSEPR Model. Journal of Chemical Education. v. 81, p. 298-304, 2004.

GIORDAN, M. O computador na educação em ciências: breve revisão crítica acerca de algumas formas de utilização. Revista Ciência e Educação, v. 11, n. 2, p. 279-304, 2005.

HARDWICKE, A. J. Using molecular models to teach chemistry. School Science Review. v. 77, n. 278, p. 59-64, 1995.

INGHAM, A. M.; GILBERT, J. K. The Use of Analogue Models by Students of Chemistry at Higher Education Level. International Journal of Science Education. v. 13, n. 2, p. 193-202, 1991.

JOHNSTONE, A. H. Why is science difficult to learn? Things are seldom what they seem. Journal of Computer Assisted Learning, n. 7, p. 75-83, 1991.

JOHNSTONE, A. H.; LETTON, K. M. Investigating undergraduate lab work. Education in Chemistry, n. 28, p. 81-83, 1990.

KIBOSS, J. K. Impact of a computer-based physics instruction program on pupils' understanding of measurement concepts and methods associated with school science. Journal of Science Education and Technology, v. 11, n. 2, p. 193-198, 2002.

KOZMA, R. B.; RUSSELL, J.; JONES, T.; MARX, N.; DAVIS, J. The use of multiple, linked representations to facilitate science understanding. In R.G.S. Vosniadou; DeCorte, E.; H. Mandel (Eds.), International perspective on the psychological foundations of technology-based learning environments. Hillsdale, NJ: Erlbaum, p. 41-60, 1996.

KRAJCIK, J. S. Developing students' understanding of chemical concepts. In S.M.Glynn, R.H. Yeany; B.K. Britton (Eds.), The psychology of learning science: International perspective on the psychological foundations of technology-based learning environments. Hillsdale, NJ: Erlbaum, p. 117-145, 1991.

LEVINE, F. S. Concepts and models. Education in Chemistry, v. 11, n. 3, p. 84-85, 1974.

MILAGRES, V. S. O. ; JUSTI, R. S. Modelos de ensino de equilíbrio químico: algumas considerações sobre o que tem sido apresentado em livros didáticos no Ensino Médio. Quí- mica Nova na Escola, n. 13, p. 41-46, 2001.

NERSESSIAN, N. J. Should physicists preach what they practice? Constructive modeling in doing and learning physics. Science and Education, v. 4, p. 203-226, 1995.

RUSSEL, J. B. Química geral. Trad. Márcia Guekezian, 3. ed., São Paulo: Pearson Makron Books. 2006. 621p.

RYLES, A. P. Teaching A-level organic chemistry mechanisms: Some suggestions. School Science Review. v. 72, n. 258, p. 71-74, 1990.

SANT’ANNA, C. M. R. Glossário de termos usados no planejamento de fármacos (recomendações da IUPAC para 1997). Química Nova, v. 25, n. 3, 2002.

SANTOS, H. F. O conceito de modelagem molecular. Cadernos temáticos de química nova na escola. São Paulo, n. 4, p. 4-5, mai, 2001.

SCHMIDT, H. J. Conceptual difficulties with isomerism. Journal of Research in Science Teaching. v. 29, n. 9, p. 995-1003, 1992.

SEDDON, G. M.; SHUBBER, K. E. The effects of color in teaching the visualization of rotation in diagrams of three dimensional structures. British Educational Research Journal. n. 11, p. 227-239, 1985.

SEDDON, G. M.; MOORE, R. G. An unexpected effect in the use of models for teaching the visualization of rotation in molecular structures. European Journal of Science Education. v. 8, p. 79-86, 1986.

SHANI, A.; SINGERMAN, A. A self-study, one paced basic organic chemistry course. Journal of Chemical Education. v. 59, p. 223-224, 1982.

SILVA, R. M. G. . Formação docente: outra lógica frente aos desafios da informatização. In: Fonseca, S. G.; Baraúna, S. M.; Miranda, A. B.. (Org.). O uno e o diverso na educação escolar. Uberlândia: EDUFU, 2005, p. 28-42.

SIMPSON, P. A new organic mythology. Education in Chemistry. v. 20, n. 5, p. 166, 1983.

TABER, K. S. Student Understanding of ionic bonding: molecular versus electrostatic framework? School Science Review, n. 78, p. 85-95, 1997.

TIBERGHIEN, A. Modeling as a basis for analyzing teachinglearning situations. Learning and Instruction, Oxford, v. 4, n. 1, p. 71-87, 1994.

TUCKEY, H., SELVARATNAM, M., BRADLEY, J., Identification and rectification of student difficulties concerning three-dimensional structures, rotation and reflection. Journal of Chemical Education, v. 68, n. 6, p. 460-464, 1991.

WHITTEN K. W.; DAVIS R. E.; PECK L.; STANLEY G. G. General Chemistry, $7^{\text {th }}$ ed., EUA: Brooks Cole, 2003. 1224p. 
WU, H., KRAJCIK, J. S., SOLOWAY, E. Promoting understanding of chemical representations: Students' use of visualization tool in the classroom. Journal of Research in Science Teaching, v. 38, n. 7, p. 821-842, 2001. 